Archives

13 | 1994

Enquêtes

\title{
Travaux pratiques d'archéologie médiévale
}

\section{Alain Guerreau}

\section{(apenEdition}

\section{Journals}

\section{Édition électronique}

URL : http://journals.openedition.org/ccrh/2706

DOI : $10.4000 /$ ccrh. 2706

ISSN : $1760-7906$

\section{Éditeur}

Centre de recherches historiques - EHESS

\section{Édition imprimée}

Date de publication : 4 octobre 1994

ISSN : 0990-9141

\section{Référence électronique}

Alain Guerreau, «Travaux pratiques d'archéologie médiévale », Les Cahiers du Centre de Recherches Historiques [En ligne], 13| 1994, mis en ligne le 27 février 2009, consulté le 20 avril 2019. URL : http:// journals.openedition.org/ccrh/2706 ; DOl : 10.4000/ccrh.2706

Ce document a été généré automatiquement le 20 avril 2019.

Article L.111-1 du Code de la propriété intellectuelle. 


\title{
Travaux pratiques d'archéologie médiévale
}

\author{
Alain Guerreau
}

1 A une demande portant sur mes expériences en matière d'archéologie médiévale, je dois bien sûr répondre d'abord en essayant de présenter celles-ci de manière aussi claire que possible, mais une telle suite d'anecdotes n'aurait guère de sens en dehors d'une réflexion critique sur ce type de pratique, aussi bien quant à ses présupposés abstraits qu'à propos des implications des conditions d'exercice professionnel de cette activité sur son développement et sur ses rapports avec l'histoire médiévale en général.

\section{Quelques expériences pratiques}

2 Jusqu'en 1983, je me contentai de quelques observations ponctuelles et rapides sur des chantiers urbains dans le centre ancien de Mâcon. A l'été 1983, mon attention fut attirée par les travaux de consolidation du pont sur la Saône (dit " pont Saint-Laurent »), travaux qui, par tranches annuelles, mirent à sec le pied des piles dans des enceintes de palplanches d'acier (batardeaux). En septembre 1983, je m'aperçus que le pied des trois piles orientales était cerné d'une considérable quantité de pieux de bois dépassant du lit de la rivière. Sur place, personne ne s'intéressait à la question. L'année suivante, je surveillai l'avancée des travaux et, dès que le lit fut à sec, je descendis et effectuai le prélèvement d'une douzaine de rondins, que je mis bientôt entre les mains de Georges Lambert, responsable de la dendrochronologie au laboratoire de chronoécologie de Besançon (CNRS). Ces prélèvements n'avaient pas été effectués correctement ; les années qui suivirent, G. Lambert vint lui-même à Mâcon. Je tentai d'accumuler le plus possible d'observations (échantillons, ramassage d'objets, photographies, mesures) lors des phases successives du chantier (1985-1986-1988). Grâce à Jean Mesqui, une série de datations ${ }^{14} \mathrm{C}$ permit d'obtenir un autre type d'information sur les pieux. Finalement, après un long temps d'incertitude et au travers de multiples difficultés, G. Lambert data un important lot de pieux de chêne de la fin du XviI ${ }^{e}$ siècle. Mais plusieurs ${ }^{14} \mathrm{C}$ et divers objets (sabots de pieux) sont médiévaux. Une synthèse de toutes les observations effectuées entre 1983 et 
1988, complétées par un examen systématique de l'iconographie ancienne, a été publiée dans deux articles, en 1989 et 1992.

3 Cette expérience me permit de mesurer les difficultés de l'intervention sur un grand chantier de travaux publics, et d'entrer en contact pratique avec l'archéométrie et ses problèmes spécifiques. Un grand pont ancien (200 mètres de long) est un ouvrage complexe, éclairé par des documents tardifs et lacunaires. L'observation directe permet de recueillir des indices importants, notamment pour les périodes anciennes, oblige à prendre en compte l'édifice en tant que structure fonctionnelle et à l'examiner dans une perspective de longue durée, ce qui n'amène cependant à des réflexions utiles que si l'on connaît suffisamment par ailleurs l'histoire générale de la région.

4 En mars 1985, après plusieurs semaines d'hésitation, j'écrivis au maire de Mâcon, pour lui indiquer qu'avant de démolir la petite église suburbaine de Saint-Clément (abandonnée par le clergé et le culte depuis une douzaine d'années), il pourrait être utile de procéder à quelques observations archéologiques. Le maire, M.-A. Rognard, répondit sur le champ et très favorablement; mon collègue et ami Christian Sapin accepta de venir organiser un sondage durant l'été. Nous découvrîmes un sarcophage, des tesselles de verre doré, des substructions romanes. Le maire engagea la ville dans un soutien massif au programme de recherche qui fut alors mis sur pied. Des campagnes annuelles se déroulèrent de 1986 à 1992, qui permirent la fouille de la plus grande partie de la zone interne au bâtiment et d'une surface extérieure assez importante. Des fondations furent dégagées en quantité notable, et plusieurs centaines de sépultures fouillées minutieusement. Des monnaies assez abondantes ( $\mathrm{du} \mathrm{IV}^{\mathrm{e}}$ au XVIII ${ }^{\mathrm{e}}$ siècle), et plus de 15000 tessons, dont une grande proportion de céramique commune grise, furent extraits du sol, ainsi que des fragments de verre, de petits objets métalliques, quelques coquilles Saint-Jacques. Dans la zone cimétériale extérieure furent reconnus plusieurs fonds de cabane. Au total, une succession enchevêtrée de bâtiments, depuis une chapelle funéraire du $\mathrm{VI}^{\mathrm{e}}$ siècle jusqu'à une église suburbaine du $\mathrm{XIX}^{\mathrm{e}}$ siècle, le tout lardé d'un indescriptible entassement de sépultures.

5 A raison de plusieurs semaines par an, j'ai donc appris sur le tas à manier la truelle, le tamis et le théodolite; à laver les tessons et à les ranger dans de petits sachets de matière plastique. J'ai amélioré mes capacités de photographe et appris à repérer les positions relatives des unités stratigraphiques. Surtout, j'ai perçu empiriquement que l'archéologie est d'abord un engagement physique; j'ai pu identifier les pesantes difficultés d'organisation pratique d'un chantier, et observer la kyrielle épuisante de démarches nécessaires aux autorisations et au financement.

6 Dans la phase d'étude du mobilier, j'ai choisi de m'intéresser aux tessons de céramique grise. Ces objets de quelques centimètres carrés, le plus souvent informes (aucun vase n'a pu être reconstitué, même partiellement) ne paraissent guère datables: production s'étendant $d u v^{e}$ au XVI ${ }^{e}$ siècle, pour le moins. J'ai constitué un échantillon de 800 tessons, répartis dans des horizons stratigraphiques bien distincts, procédé à une observation méthodique à la loupe binoculaire et noté un certain nombre de caractères de couleur et de fréquence s'agissant de la pâte et des nombreuses inclusions qu'elle renferme. Un traitement statistique complexe (et long) a abouti à la définition de classes paraissant correspondre à des phases successives.

7 A l'heure actuelle, en collaboration avec un collègue géologue, je tente une définition chimique fine des divers composants, de la pâte et des inclusions (au moyen d'un 
microscope électronique à balayage, au Museum). Des traces surprenantes de manganèse et de potassium ont été repérées, qui conduisent à s'interroger sur la nature de certaines inclusions. Ultérieurement est prévu un programme de datation de quelques échantillons par thermoluminescence. Il n'est pas exclu que l'on puisse parvenir à des conclusions originales sur l'évolution des techniques de fabrication de la céramique commune médiévale (en Bourgogne du Sud tout au moins). Expérience concrète de traitement d'un élément de mobilier très abondant, mais peu loquace.

Je pourrais encore ranger parmi mes activités archéologiques des pratiques d'observation matérielle méthodiques, quoiqu'extérieures à toute fouille.

Lors de mes travaux dans les archives communales de Mâcon des XIV $-\mathrm{XVI}^{\mathrm{e}}$ siècles, j'ai relevé systématiquement le filigrane de tous les papiers examinés.

Dans le cadre d'une entreprise de datation relative des dizaines de petites églises romanes de la région de Cluny-Tournus-Mâcon, j'ai mesuré, avec ma femme, une vingtaine de ces petites églises (plusieurs centaines de mesures, en plan et en élévation). Ce qui m'a permis bien sûr d'établir des plans précis (jusqu'alors inexistants) mais aussi de mieux saisir la logique de structuration de ces bâtiments et de passer à une analyse métrologique de ces édifices, c'est-à-dire à une tentative de détermination des unités de mesure employées au moment de leur construction. Certaines conclusions générales issues de ces recherches ont été utilisées comme hypothèse pour l'examen de bâtiments de grande taille et ont abouti au repérage des modules utilisés; d'où il ressort, à l'inverse de ce que l'on aurait pu imaginer, une quasi-stabilité des unités de mesure employées dans les constructions ecclésiastiques du $v^{e}$ au début du XII ${ }^{e}$ siècle.

\section{L'interprétation historique}

11 Dans un premier temps de réflexion, il me semble nécessaire de distinguer trois phases empiriques, présentant chacune des difficultés propres.

\section{La fouille stricto sensu}

12 Si l'on fréquente durant quelques semaines deux ou trois chantiers bien organisés et bien conduits, et que l'on a affaire à des responsables doués d'un minimum de pédagogie, on a appris l'essentiel. On doit noter que cet apprentissage de base est indispensable, car les méthodes pratiques ont réalisé de sensibles progrès durant les dernières décennies; on parvient ainsi, par exemple, à repérer les traces des anciennes structures en bois, ce qui autorise une collecte étonnante qui est en train de modifier notre vision de l'occupation du sol durant le haut Moyen Age. Il reste que l'on s'habitue aussi vite à la fouille qu'à la lecture des écritures anciennes.

\section{L'étude des objets}

13 L'approche élémentaire (relevé, mesure, photographie) s'apprend très vite. Mais les examens qui doivent déboucher sur des identifications ou des caractérisations précises (ossements humains et animaux, pollens, graines, charbons de bois, mortiers, monnaies) supposent une formation approfondie, beaucoup plus longue à acquérir, et dont la mise en œuvre peut réclamer beaucoup de temps. Les analyses physico-chimiques qui 
aboutissent à des caractérisations fines et surtout à des datations nécessitent à la fois une compétence et des installations de laboratoire appropriées. Si une certaine spécialisation est inévitable, la parcellisation des préoccupations aboutit à une catastrophique déperdition d'information et à des formes d'interprétation d'une indigence à pleurer.

\section{L'interprétation historique}

Le point crucial est ici : l'ensemble des observations archéologiques, c'est-à-dire portant sur des objets, n'a de sens qu'à partir du moment où lesdites observations peuvent être intégrées au système global des connaissances relatives à la société qui a produit ces objets. « Interprétation archéologique » est un syntagme absolument vide. Extraire du sol des poteries, des squelettes ou des graines n'est qu'un passe-temps futile si cette pratique n'est pas intégrée à une démarche dont la finalité est l'amélioration des connaissances concernant telle ou telle société passée. On ne peut plus admettre (même lorsqu'il s'autoproclame "nouvelle archéologie ») l'état d'esprit antiquaire, au sens du xix siècle, qui se préoccupe des objets anciens pour eux-mêmes, refusant la seule catégorie acceptable, celle de document. C'est la société qui donne leur sens aux objets, en aucun cas l'inverse.

On ne peut cependant pas éviter d'observer que d'assez nombreux collègues qui fouillent, et s'autodéfinissent « archéologues », confondant la fin et les moyens, consacrent très peu d'effort à ce troisième aspect, s'arrêtant de fait à la considération des objets pour euxmêmes, les questions de datation apparaissant alors comme fin ultime. D'où une invraisemblable surestimation de la notion de "phase ", le moindre site donnant lieu à une segmentation presque infinie (phase Ia1, Ia2, Ib, etc...) qui permet d'éviter de se poser toute question sur la relation entre les objets et la société et, a fortiori, de s'interroger sur ce que ces objets peuvent nous apprendre de la société considérée, de son fonctionnement et de sa dynamique.

Dans cette perspective, on ne peut que rejeter l'artefact que constitue la notion de "culture matérielle ». A-t-on déjà rencontré la notion de «culture spirituelle» ou de «culture idéelle » qui en constitue le complément logique inévitable? Considérons la paléocarpologie. Une fouille met en évidence, sur un site rural donné, une répartition des céréales où domine le seigle (par rapport au froment), ou l'épeautre, que sais-je encore. En vérité, la question de savoir ce que mangeaient les habitants n'a, en soi, qu'une valeur anecdotique (le vieux genre «vie quotidienne » n'est qu'un kaléidoscope déprimant, qui ne mérite aucune réhabilitation). En revanche, les questions de choix d'une technique agricole et de valorisation de telle ou telle consommation, nécessairement imbriquées, renvoient autant l'une que l'autre à une recherche sur les structures sociales; là, il ne s'agit plus de collectionner des ronds de bière, mais de confronter des éléments concrets à des hypothèses abstraites, de manière à faire faire progresser à la fois le réalisme et la rationalité de nos connaissances sur une société.

17 L'étude de la taille des bâtiments amène à des réflexions analogues. L'observation conduit à relever les dimensions de certains objets (murs, surfaces). Si l'objet est le produit d'une construction (activité sociale par excellence), il est pour le moins raisonnable de faire l'hypothèse que les constructeurs disposaient, au moins mentalement, d'un certain plan au moment d'entamer cette construction, et que la fixation au sol de ce plan supposait un minimum d'activité de mesure. La dimension de l'objet est un caractère concret directement perceptible; la restitution des pratiques de mesure (qui prend appui sur 
l'analyse de ces dimensions) fait appel à une série de notions abstraites liées à certaines structures sociales. Le fait d'avoir soi-même mesuré l'objet et d'en avoir une perception matérielle directe (ou d'avoir mesuré des objets analogues) est, en pratique, une condition presque nécessaire à la construction d'un raisonnement métrologique approprié, mais sûrement pas une condition suffisante.

Je dois confesser que j'éprouve une extrême réticence chaque fois que je lis l'expression rituelle " données historiques et archéologiques... ». La liturgie funéraire serait donc une « donnée historique » et les squelettes des « données archéologiques »; la production de céramiques, une " donnée historique » et les tessons une "donnée archéologique ». Une telle distinction traduit une irrecevable simplification intellectuelle On doit toujours en revenir à une considération extrêmement simple, quoique systématiquement perdue de vue toute structure sociale est à la fois un ensemble de relations abstraites et une réalité matérielle.

\section{L'archéologie : une recherche historique de terrain}

19 La revendication, aigre et irrationnelle, d'autoproclamation de «l'archéologie » comme activité autocentrée et indépendante de l'histoire, est avant tout le produit d'une situation pratique détestable, qu'il serait urgent de réformer.

Le lien, au sein de l'Université, entre "l'histoire de l'art » et "l'archéologie » est un archaïsme insoutenable, aux effets délétères. L'essentiel de ce qu'on dénomme aujourd'hui «archéologie» est une activité purement historique (sauf s'agissant de histoire...) qui n'a avec « l'histoire de l'art » que des relations épisodiques et distendues. Une formation, même excellente, dans ce dernier domaine n'apporte rien, sinon des préjugés, à qui prétend fouiller et interpréter convenablement le produit de ses fouilles.

21 Il serait par conséquent raisonnable d'intégrer «l'archéologie », comme pratique «de terrain", au cursus obligatoire des historiens et, en retour, de ne confier les fouilles importantes qu'à des responsables ayant reçu une formation historique très solide. Il faut faire disparaître la distinction extraordinairement nuisible entre « homme d'archives » et "homme de terrain ", en parvenant à ce que tous les médiévistes conjuguent les deux approches. Chacun sait très bien qu'on n'utilise pas sans risques graves des documents dont la matérialité est inconnue ; chacun sait que, pour éviter de grossières erreurs dans la lecture des textes édités, il faut au moins avoir eu un contact approfondi avec des chartes originales, des registres, des manuscrits; comment imaginer qu'un médiéviste puisse réellement et intelligemment tirer parti d'informations provenant de fouilles s'il n'a jamais tenu une truelle?

La tutelle administrative de l'ensemble des activités de fouille en France par la Direction du Patrimoine du Ministère de la Culture est plus que problématique. Car si les archives ou les bibliothèques relèvent assez logiquement de cette direction en tant que ces services ont une activité prioritaire de conservation, il tombe sous le sens que le fouilleur, par définition, fait disparaitre les couches de terrain qu'il prend comme objet d'observation. La fouille est fondamentalement une activité de recherche et non de conservation. L'existence d'un important corps de "conservateurs de l'archéologie ", intégré dans les cadres du Ministère de la Culture, aux préoccupations gestionnaires et jugé sur de tout autres critères que ceux de la contribution à l'avancement des 
connaissances historiques, est une situation malsaine, en dépit des cas individuels, nombreux, qui méritent tous les éloges.

23 Les sites médiévaux fouillés sont légion. Seule la réflexion historique peut donner sens et cohérence à l'énorme moisson d'informations qui en ressort, le plus souvent en vrac. Les historiens sont au pied du mur: la réalité n'est pas faite que de papier, la terre est là aussi. 